\title{
Reduction in Heart Rate Variability in Autosomal Dominant Polycystic Kidney Disease
}

\author{
Silvia Lai ${ }^{a}$ Marco Mangiullia Adolfo M. Perrotta ${ }^{a}$ \\ Gianluca Di Lazzaro Giraldi $^{b}$ Massimo Testorio $^{c}$ Edoardo Rosato $^{b}$ \\ Rosario Ciancia Antonietta Gigante ${ }^{b}$ \\ a Nephrology Unit, Department of Translational and Precision Medicine, Sapienza University \\ of Rome, Rome, Italy; ${ }^{b}$ Department of Translational and Precision Medicine, Sapienza \\ University of Rome, Rome, Italy; ${ }^{C}$ Nephrology Unit, Department of Gynecologic-Obstetrical \\ Sciences and Urological Sciences, Sapienza University of Rome, Rome, Italy
}

\section{Keywords}

Autosomal dominant polycystic kidney disease - Cardiovascular disease · Hypertension . Heart rate variability

\begin{abstract}
Introduction: Cardiovascular disease is one of the main causes of morbidity and mortality in patients with autosomal dominant polycystic kidney disease (ADPKD). Autonomic dysfunction is associated with an increased risk for all cardiovascular events in the general population and can be evaluated with heart rate variability (HRV). Objective: To evaluate HRV in ADPKD patients with mild hypertension versus hypertensive patients with organ damage and healthy controls (HC). Materials and Methods: We have enrolled 65 patients: 21 ADPKD patients (10 males), 20 patients with hypertension (14 males), and $24 \mathrm{HC}$ (10 males). Biochemical analysis, clinical evaluation, anthropometric data, intima-media thickness, 24-h ECG Holter recording, and echocardiography were investigated at the time of enrollment. Results: No significant differences in HRV parameters were found between ADPKD with mild hypertension and hypertensive patients with organ damage. The median of HRV variables in time domain as SDNN (global autonomic activity) was significantly lower in ADPKD and hypertensive patients than $\mathrm{HC}(p<0.05)$. In the frequency domain analysis, low frequency $(\mathrm{LF})$, which mainly reflects the sympathetic component, showed higher values in ADPKD and hypertensive patients than HC during the night $(p<0.01)$. During the night, the sympathovagal balance, LF/high frequency $(\mathrm{HF})$, showed higher values in ADPKD and hypertensive patients than HC $(p<0.0001)$. Conversely, LF day was lower in ADPKD and hypertensive patients than $\mathrm{HC}(p<0.01)$. HF, which
\end{abstract}


mainly reflects the parasympathetic component, was lower in ADPKD and hypertensive patients during the night than $\mathrm{HC}(p<0.0001)$. Conclusions: HRV reduction is present in ADPKD patients with mild hypertension in the absence of organ damage. The evaluation of sympathovagal balance can provide novel information on the cardiovascular risk in ADPKD patients in addition to classical risk factors.

(C) 2019 The Author(s)

Published by S. Karger AG, Basel

\section{Introduction}

Autosomal dominant polycystic kidney disease (ADPKD) is a monogenic hereditary disease characterized by multiple, bilateral cystic dilatation of the renal tubules. By the age of $50-60$ years $[1,2], 50 \%$ of patients undergo a gradual subversion of the renal parenchyma with an enlargement in the total kidney volume and eventually reach end-stage renal disease. Cardiovascular complications are the major cause of morbidity and mortality, with a cardiacrelated death that is estimated to be 1.6- to 3.2-fold higher in these patients than in the general population [3, 4]. Hypertension, endothelial dysfunction, systemic inflammation, and accelerated atherosclerosis are alterations found at a very early stage of the disease and are responsible for increasing both cardiovascular risks and progression towards end-stage renal disease. Hypertension is very common and often diagnosed before the onset of renal failure in 50-75\% of ADPKD patients. The pathogenesis of hypertension in ADPKD is not yet fully elucidated, but some mechanisms have been revealed, such as activation of the reninangiotensin-aldosterone system (RAAS), impaired nitric oxide-related vasorelaxation, increased sympathetic nerve activity, increased plasma endothelin-1 concentration, and insulin resistance [5]. Autonomic dysfunction (AD) represents a cardiovascular risk factor that increases morbidity and mortality among patients. Autonomic activity can be evaluated with heart rate variability (HRV) [6]. In physiological conditions, dependence of HRV on heart rate is determined by the autonomic nervous system. In the course of pathological conditions such as myocardial ischemia, a reduction in parasympathetic control leads to sympathetic hyperactivity with cardiac electrical instability and left ventricular dysfunction [7].

Recently, Chou et al. [8] have demonstrated that HRV represents a predictor of rapid kidney injury in chronic kidney disease patients on dialysis. They found that AD depends on the stage of chronic kidney disease and that the lowest HRV is present in end-stage renal disease. In essential hypertension, an imbalance has been reported between the sympathetic and parasympathetic systems [9]. To date, only one study has been conducted to evaluate heart rate recovery (HRR) in ADPKD patients. In 28 ADPKD patients without hypertension, Orscelik et al. [10] showed impaired HRR suggesting a link between ADPKD and the autonomic nervous system. The aim of the present study was to evaluate HRV, using time and frequency domain, in ADPKD patients with mild hypertension versus hypertensive patients with organ damage and healthy controls (HC).

\section{Materials and Methods}

We have enrolled 65 patients from April to December 2018: 21 ADPKD patients (10 males), 20 patients with hypertension and organ damage (14 males), and $24 \mathrm{HC}$ (10 males). Biochemical analysis, clinical evaluation, anthropometric data, intima-media thickness, 24-h ECG Holter recording, and echocardiography were investigated at the time of enrollment. ADPKD patients with established cardiovascular disease like myocardial infarction, stroke, presence of atheromatous plaques on imaging, peripheral artery disease, and atrial fibril- 


\section{Kidney \\ Blood Pressure \\ Research}

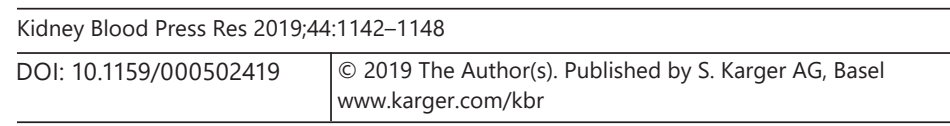

Lai et al.: Heart Rate Variability Reduction in Autosomal Dominant Polycystic Kidney Disease

Table 1. Anthropometric and clinical features of patients enrolled

\begin{tabular}{|c|c|c|c|}
\hline & ADPKD patients & Hypertensive patients & Healthy controls \\
\hline Females/males & $11 / 10$ & $6 / 14$ & $14 / 10$ \\
\hline Median age, years & $43(29-66)$ & $47(31-63)$ & $47(41-65)$ \\
\hline Body mass index, $\mathrm{kg} / \mathrm{m}^{2}$ & $23(18.8-26)$ & $27(22-35)$ & $23(20-29)$ \\
\hline cIMT, mm & $0.68(0.60-0.90)$ & $1(0.9-1.9)$ & $0.61(0.60-0.71)$ \\
\hline \multicolumn{4}{|l|}{ Blood pressure, mm Hg } \\
\hline Systolic & $130(110-140)$ & 135 (120-177) & $112(95-128)$ \\
\hline Diastolic & $80(70-90)$ & $85(70-100)$ & $65(55-81)$ \\
\hline Triglycerides, mg/dL & $88(71-138)$ & $88(34-250)$ & $81(45-118)$ \\
\hline $\mathrm{HDL}, \mathrm{mg} / \mathrm{dL}$ & $52(35-90)$ & $49(41-76)$ & $52(40-65)$ \\
\hline $\mathrm{LDL}, \mathrm{mg} / \mathrm{dL}$ & $130(73-157)$ & $133(87-176)$ & $119(96-138)$ \\
\hline Total cholesterol, mg/dL & 201 (148-237) & $203(168-245)$ & $0.9(0.8-1.2)$ \\
\hline Serum creatinine, $\mathrm{mg} / \mathrm{dL}$ & $1.25(0.8-4)$ & $0.9(0.8-1.09)$ & $0.8(0.7-1.07)$ \\
\hline \multicolumn{4}{|l|}{ Patients, $n$} \\
\hline Myocardial infarction & 0 & 1 & 0 \\
\hline Stroke & 0 & 1 & 0 \\
\hline Carotid plaques & 0 & 3 & 0 \\
\hline Ejection fraction, \% & $65(60-65)$ & $58(55-65)$ & $65(60-68)$ \\
\hline
\end{tabular}

cIMT, carotid intima-media thickness; HDL, high-density lipoprotein; LDL, low-density lipoprotein.

lation were excluded. Others exclusion criteria, also for the hypertensive group, were diabetes, valvular abnormalities, arrhythmias, thyroid dysfunction, severe infections, malignancies, chronic liver disease, and chronic respiratory obstruction.

At the time of enrollment, none of the ADPKD and hypertensive patients were treated with $\beta$-blockers, antiarrhythmic drugs, ACE inhibitors, or angiotensin receptor blockers. Subjects' written consent was obtained. The study was conducted according to the criteria set by the Declaration of Helsinki and received approval of the local ethics committee.

\section{Heart Rate Variability}

All subjects underwent 24-h ambulatory 3-channel ECG Holter recording (Lifecard CF; Spacelabs Healthcare, Snoqualmie, WA, USA). HRV is a noninvasive marker to evaluate autonomic nervous system activity in time and frequency domain, according to the recommendation of the European Society of Cardiology and the North American Society of Pacing and Electrophysiology [11]. At 10-min intervals, all the patients were analyzed with ECG recording, and the time registration was divided into 2 periods: day (7 a.m. to 12 p.m.) and night (12 p.m. to 7 a.m.). Artificial and arrhythmic data were excluded. In the time domain, we have evaluated the standard deviation of normal-to-normal RR intervals (SDNN) (ms) and the square root of the mean of the sum of the squares of differences between adjacent NN intervals (RMSSD) that represent markers of the global sympathetic and the parasympathetic system, respectively. In the frequency domain, fast Fourier transform was used to obtain power spectral estimates of HRV to assess low frequency (LF: 0.04-0.15 Hz, modulated mainly by the sympathetic system) and high frequency (HF: $0.15-0.40 \mathrm{~Hz}$, modulated by the parasympathetic system) in normalized units. The LF/HF ratio represents the sympathovagal balance. Data analyses were performed with software (Accuplus 363; Del Mar Avionics, Irvine, CA, USA)

\section{Statistical Analysis}

Data management and analysis were performed using IBM ${ }^{\circledR}$ SPSS $^{\circledR}$ statistics 25.0 for Windows ${ }^{\circledR}$ (IBM Corporation, Armonk, NY, USA). The normality of variables was tested by the 


\section{Kidney \\ Blood Pressure \\ Research}

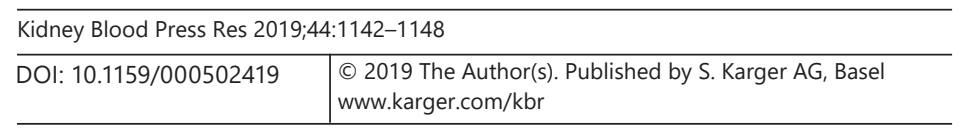

Lai et al.: Heart Rate Variability Reduction in Autosomal Dominant Polycystic Kidney Disease

Table 2. Parameters of heart rate variability in ADPKD patients, hypertensive patients, and healthy controls

\begin{tabular}{lcccl}
\hline & ADPKD patients & Hypertensive patients & Healthy controls & $p$ value \\
\hline SDNN, ms & $125.7(119.3-148.5)$ & $130.2(118-149)$ & $153(130.9-173.9)$ & $<0.05$ \\
RMSSD, ms & $37.7(27.6-46.9)$ & $31.1(28.1-43.4)$ & $38(34.5-46.9)$ & $>0.05$ \\
LF nu day & $56(51-60.7)$ & $60(56.5-63.1)$ & $63.8(60.9-66.1)$ & $<0.01$ \\
HF nu day & $34(32-40)$ & $32(26.4-35)$ & $30.5(26.9-33.4)$ & $>0.05$ \\
LF/HF & $1.9(1.6-2.6)$ & $2(1.8-2.5)$ & $1.7(1.6-2.2)$ & $>0.05$ \\
LF night, nu & $51.6(41.8-58)$ & $53(49-58)$ & $43.2(39.8-49.8)$ & $<0.01$ \\
HF night, nu & $38.8(33.5-49)$ & $39(38-48)$ & $50.7(43.8-56.4)$ & $<0.0001$ \\
LF/HF night & $1.7(1.5-2.3)$ & $1.6(1.5-2.6)$ & $0.9(0.8-1.2)$ & $<0.0001$ \\
HR, bpm & $79.5(71.3-87.9)$ & $79.9(74-81)$ & $74.9(70.3-81)$ & $>0.05$ \\
\hline
\end{tabular}

SDNN, standard deviation of normal-to-normal RR intervals; RMSSD, root mean square of successive differences; LF, low frequency; HF, high frequency; nu, normal units; night, 0-7 h; day, 7-24 h; HR, heart rate.

Shapiro-Wilk normality test. All continuous normally distributed variables were expressed as means \pm SD. Nonnormally distributed variables were described as medians and confidence intervals. The difference between numerical data was analyzed using nonparametric tests (Kruskal-Wallis test). Bivariate correlations and the degree of association between the variables were obtained with the Spearman test. Differences between qualitative variables were assessed by the Fisher exact test. A value of $p<0.05$ was considered statistically significant. The Student $t$ test was used to determine differences between the groups.

\section{Results}

Table 1 shows the anthropometric and clinical features of patients enrolled. No significant differences in HRV parameters were found between ADPKD with mild hypertension and hypertensive patients with organ damage. Table 2 shows HRV parameters in ADPKD, hypertensive patients, and HC. LF night showed a trend to higher values in ADPKD and hypertensive patients compared to HC $(p<0.01)$ (Fig. 1A). LF/HF night demonstrated high values in ADPKD and hypertensive patients compared to HC ( $p<0.0001)$ (Fig. 1B). Conversely, LF day was lower in ADPKD and hypertensive patients than HC $(p<0.01)$. HF night was also decreased in ADPKD and hypertensive patients compared to HC $(p<0.0001)$ (Fig. 1C). The median of HRV variables in time domain as SDNN (global autonomic activity) was significantly lower in ADPKD and hypertensive patients than HC $(p<0.05)$ (Fig. 1D).

\section{Discussion}

In the present study, ADPKD patients reported statistical differences in the global autonomic activity represented by SDNN versus HC. In fact, when compared to HC, ADPKD patients showed a lower SDNN, much like that reported in hypertensive patients with organ damage. Previous studies reported that patients with SDNN $<50 \mathrm{~ms}$ after myocardial infarction have an increased risk of mortality at follow-up when compared to patients with SDNN >100 ms [12]. Worldwide, essential hypertension represents the major cause of cardiovascular disease and death [13]. Several studies demonstrated AD in essential hypertension. The pathogenesis of hypertension is multifactorial, but a high sympathetic tone is activated in the early stages of essential hypertension. Thus, an imbalance between the 


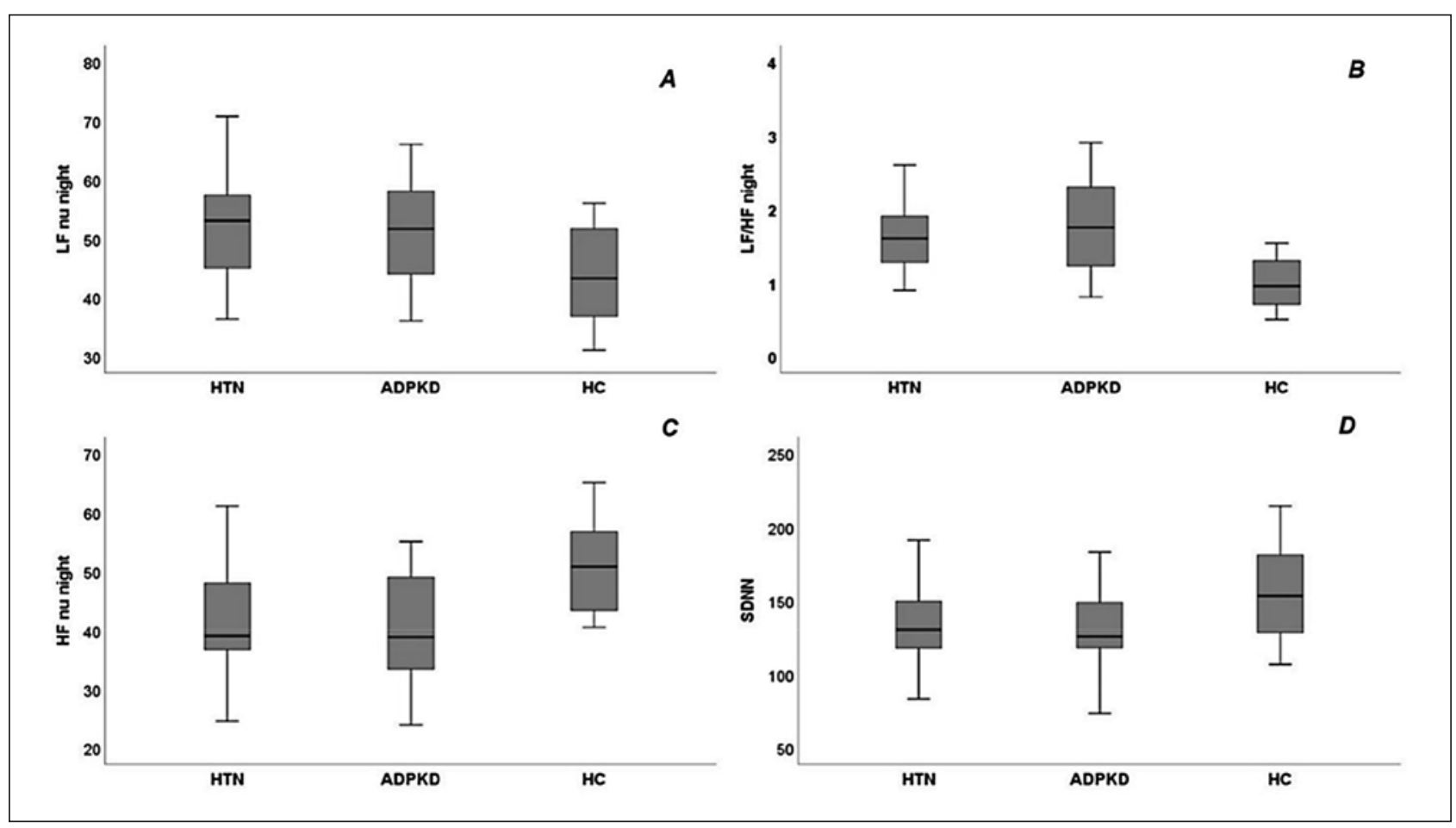

Fig. 1. Low frequencies (LF) nu night (A), LF/high frequency (HF) night (B), HF nu night (C), and standard deviation of normal-to-normal RR intervals (SDNN; D) in hypertensive patients (HTN), patients with autosomal dominant polycystic kidney disease (ADPKD), and healthy controls (HC). nu, normal units.

sympathetic and parasympathetic tones is present both in the early and advanced stages of the disease characterized by organ damage. It has been hypothesized that high arterial stiffness due to elevated blood pressure can reduce baroreflex sensitivity increasing the sympathetic tone [14]. The pathogenesis of hypertension related to ADPKD differs from essential hypertension. The RAAS is more activated in ADPKD patients with hypertension than in patients with essential hypertension. Hypertension related to ADPKD occurs early and is due to cyst enlargement that causes renal ischemia with renin release, complicated by endothelial dysfunction, reduced nitric oxide, and sympathetic tone activation [15]. Considering that in ADPKD continuous RAAS stimulation worsens hypertension and accelerated cyst growth, it is not surprising to find in our study high LF and LF/HF levels, confirming, together with SDNN reduction a sympathetic prevalence. Furthermore, an imbalance between the sympathetic and parasympathetic tones has been detected, characterized by an abnormal LF/HF ratio especially during the night, with higher sympathetic tone due to circadian rhythm loss, while the HF component does not show the expected physiological increase. Cerasola et al. [16] found higher catecholamine levels in 30 hypertensive ADPKD patients than in 50 essential hypertensive patients. The authors suggest that increased activity of the sympathetic system could play a role in the pathogenesis of hypertension associated with ADPKD.

However, assessment of muscle sympathetic nerve activity, measured directly from the peroneal nerve by microneurography, is considered the gold standard to evaluate autonomic activity [17]. In a previous study, Orscelik et al. [10] already evaluated autonomic activity between normotensive ADPKD patients and HC. The authors demonstrated that impaired HRR index was correlated with normotensive ADPKD patients. Although in this study ADPKD patients were normotensive, the authors suggested an association with AD 


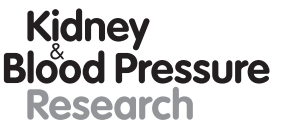

Research

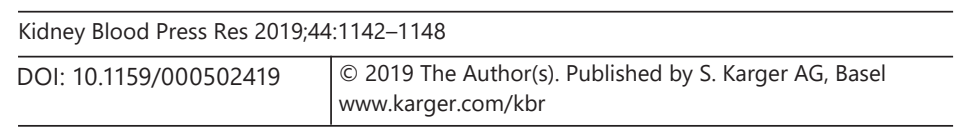

Lai et al.: Heart Rate Variability Reduction in Autosomal Dominant Polycystic Kidney Disease

and ADPKD in the early stage of the disease. Autonomic function can be studied through HRV, HRR index, and systolic blood pressure variability. HRR is a simple tool for assessing the reduction in heart rate after the cessation of exercise and is related to parasympathetic activity. HRV refers to a variation in the beat-to-beat interval and measures the time and frequency domain able to evaluate indirectly the global sympathetic and parasympathetic system with sympathovagal balance. Thus, as previously shown in the literature, HRV parameters seem to be more sensitive than HRR to reveal increased sympathetic tone [18]. In our study, patients with ADPKD present the same HRV parameters as those found in essential hypertension with organ damage, such as myocardial infarction or stroke, for example. The essential hypertension group showed a median intima-media thickness of 1 $\mathrm{mm}(0.9-1.9)$, and 3 of them showed carotid artery stenosis. In the characteristics of hypertension mediated by organ damage, the presence of carotid plaques automatically reclassifies patients from intermediate to high risk in developing cardiovascular disease [13]. It has been hypothesized that renal bilateral involvement with ischemia and RAAS stimulation in ADPKD has a pathogenesis compatible with bilateral atherosclerotic renal artery stenosis $[19,20]$. Recently, AD with low SDNN has been demonstrated also in atherosclerotic renal artery stenosis compared to $\mathrm{HC}$. The authors suggest that inflammation could play a role in the pathway between autonomic tone and atherosclerosis [21]. In our study, no statistical differences were found between HRV parameters and renal function. Therefore, we believe that in ADPKD, hypertension related to cyst enlargement and RAAS activation could only worsen the autonomic system primarily damaged by the underlying disease.

Limitation of the Study

The limitations of our study are the relatively small cohort of hypertensive and ADPKD patients, and the cross-sectional, single-center design. Large studies with a high number of patients are needed to confirm the reported data.

\section{Conclusions}

We showed a significant reduction in HRV in ADPKD patients with respect to the HC. The 24-h ECG Holter with evaluation of HRV is one of the noninvasive tools to evaluate autonomic activity in predicting cardiovascular events. We suggest to include HRV assessment in association with traditional risk factors at an early stage of the disease to better stratify cardiovascular risk in ADPKD patients.

\section{Statement of Ethics}

All procedures performed in studies involving human participants were in accordance with the ethical standards of the institutional and/or national research committee at which the studies were conducted and with the 1964 Helsinki Declaration and its later amendments or comparable ethical standards. Institutional Review Board approval has been obtained. Informed consent was obtained from all individual participants included in the study.

\section{Disclosure Statement}

The authors have declared that no conflict of interest exists. 


\section{Kidney \\ Blood Pressure Research}

\begin{tabular}{l|l}
\hline Kidney Blood Press Res 2019:44:1142-1148 \\
\hline DOI: 10.1159/000502419 & $\begin{array}{l}\text { @ 2019 The Author(s). Published by S. Karger AG, Basel } \\
\text { www.karger.com/kbr }\end{array}$ \\
\hline
\end{tabular}

Lai et al.: Heart Rate Variability Reduction in Autosomal Dominant Polycystic Kidney Disease

\section{Funding Sources}

This study was not funded.

\section{Author Contributions}

The authors alone are responsible for the content and writing of the paper. The manuscript has been seen and approved by all authors.

\section{References}

1 Watnick T, Germino GG. Molecular basis of autosomal dominant polycystic kidney disease. Semin Nephrol. 1999 Jul;19(4):327-43.

2 Loghman-Adham M, Soto CE, Inagami T, Cassis L. The intrarenal renin-angiotensin system in autosomal dominant polycystic kidney disease. Am J Physiol Renal Physiol. 2004 Oct;287(4):F775-88.

3 He WB, Xiao WJ, Tan YQ, Zhao XM, Li W, Zhang QJ, et al. Novel mutations of PKD genes in Chinese patients suffering from autosomal dominant polycystic kidney disease and seeking assisted reproduction. BMC Med Genet. 2018 Oct;19(1):186.

4 Ecder T. Cardiovascular complications in autosomal dominant polycystic kidney disease. Curr Hypertens Rev. 2013 Feb;9(1):2-11.

5 Luciano RL, Dahl NK. Extra-renal manifestations of autosomal dominant polycystic kidney disease (ADPKD): considerations for routine screening and management. Nephrol Dial Transplant. 2014 Feb;29(2):247-54.

6 Simula S, Vanninen E, Lehto S, Hedman A, Pajunen P, Syvänne M, et al. Heart rate variability associates with asymptomatic coronary atherosclerosis. Clin Auton Res. 2014 Feb;24(1):31-7.

7 Li HR, Lu TM, Cheng HM, Lu DY, Chiou CW, Chuang SY, et al. Additive Value of Heart Rate Variability in Predicting Obstructive Coronary Artery Disease Beyond Framingham Risk. Circ J. 2016;80(2):494-501.

8 Chou YH, Huang WL, Chang CH, Yang CCH, Kuo TBJ, Lin SL, et al. Heart rate variability as a predictor of rapid renal function deterioration in chronic kidney disease patients. Nephrology (Carlton). 2019 Aug;24(8):806813.

9 Erdogan D, Gonul E, Icli A, Yucel H, Arslan A, Akcay S, et al. Effects of normal blood pressure, prehypertension, and hypertension on autonomic nervous system function. Int J Cardiol. 2011 Aug; 151(1):50-3.

10 Orscelik O, Kocyigit I, Baran O, Kaya C, Dogdu O, Zengin H, et al. Impairment of heart rate recovery index in autosomal-dominant polycystic kidney disease patients without hypertension. Blood Press. 2012 Oct;21(5): $300-5$.

11 Heart rate variability: standards of measurement, physiological interpretation and clinical use. Task Force of the European Society of Cardiology and the North American Society of Pacing and Electrophysiology. Circulation. 1996 Mar;93(5):1043-65.

12 Huikuri HV, Stein PK. Heart rate variability in risk stratification of cardiac patients. Prog Cardiovasc Dis. 2013 Sep-Oct;56(2):153-9.

13 Williams B, Mancia G, Spiering W, Agabiti Rosei E, Azizi M, Burnier M, et al.; ESC Scientific Document Group. 2018 ESC/ESH Guidelines for the management of arterial hypertension. Eur Heart J. 2018 Sep;39(33):3021104.

14 Carthy ER. Autonomic dysfunction in essential hypertension: A systematic review. Ann Med Surg (Lond). 2013 Dec;3(1):2-7.

15 Chapman AB, Johnson A, Gabow PA, Schrier RW. The renin-angiotensin-aldosterone system and autosomal dominant polycystic kidney disease. N Engl J Med. 1990 Oct;323(16):1091-6.

16 Cerasola G, Vecchi M, Mulè G, Cottone S, Mangano MT, Andronico G, et al. Sympathetic activity and blood pressure pattern in autosomal dominant polycystic kidney disease hypertensives. Am J Nephrol. 1998;18(5): 391-8.

17 Macefield VG. Sympathetic microneurography. Handb Clin Neurol. 2013;117:353-64.

18 Zdrenghea D, Sitar-Tăut A, Pop D. Comparison between heart rate variability and recovery in ischemic patients. Rom J Intern Med. 2007;45(2):171-5.

19 Ecder T, Schrier RW. Hypertension in autosomal-dominant polycystic kidney disease: early occurrence and unique aspects. J Am Soc Nephrol. 2001 Jan;12(1):194-200.

20 Miller AJ, Arnold AC. The renin-angiotensin system in cardiovascular autonomic control: recent developments and clinical implications. Clin Auton Res. 2019 Apr;29(2):231-43.

21 Gigante A, Zingaretti V, Proietti M, Rosato E, Cianci R, HRV Study Group. Autonomic dysfunction and cardiovascular risk in patients with atherosclerotic renal artery stenosis: A pilot study. Eur J Intern Med. 2018 Jun; 52:e19-21. 